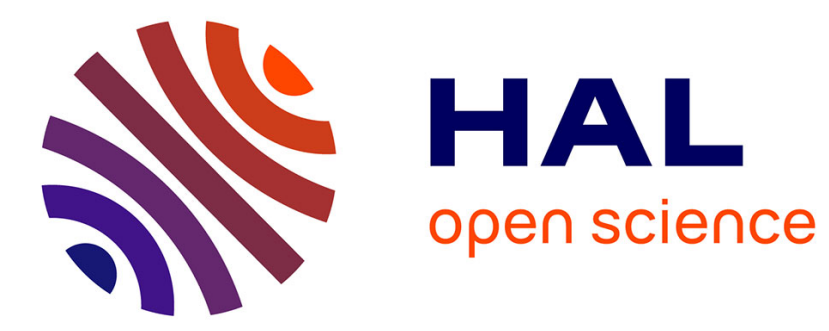

\title{
Towards Thermal Reading of Magnetic States in Hall Crosses
}

\author{
Y. Xu, S. Petit-Watelot, V. Polewczyk, G. Parent, F. Montaigne, J.-E. \\ Wegrowe, S. Mangin, D. Lacroix, M. Hehn, D. Lacour
}

\section{- To cite this version:}

Y. Xu, S. Petit-Watelot, V. Polewczyk, G. Parent, F. Montaigne, et al.. Towards Thermal Reading of Magnetic States in Hall Crosses. Physical Review Applied, 2018, 9, pp.034028. 10.1103/PhysRevApplied.9.034028 . hal-01814995

\section{HAL Id: hal-01814995 \\ https://hal.science/hal-01814995}

Submitted on 29 Jun 2018

HAL is a multi-disciplinary open access archive for the deposit and dissemination of scientific research documents, whether they are published or not. The documents may come from teaching and research institutions in France or abroad, or from public or private research centers.
L'archive ouverte pluridisciplinaire HAL, est destinée au dépôt et à la diffusion de documents scientifiques de niveau recherche, publiés ou non, émanant des établissements d'enseignement et de recherche français ou étrangers, des laboratoires publics ou privés. 


\title{
Towards Thermal Reading of Magnetic States in Hall Crosses
}

\author{
Y. Xu, ${ }^{1}$ S. Petit-Watelot, ${ }^{1}$ V. Polewczyk, ${ }^{1}$ G. Parent, ${ }^{2}$ F. Montaigne, ${ }^{1}$ J.-E. Wegrowe, ${ }^{3}$ \\ S. Mangin, ${ }^{1}$ D. Lacroix, ${ }^{2}$ M. Hehn, ${ }^{1}$ and D. Lacour ${ }^{1, *}$ \\ ${ }^{1}$ Institut Jean Lamour, CNRS UMR 7198, Université de Lorraine, Vandœeuvre-lès-Nancy F-54506, France \\ ${ }^{2}$ Université de Lorraine, LEMTA, CNRS-UMR 7563, Vandoeuvre-lès-Nancy F-54500, France \\ ${ }^{3}$ Ecole Polytechnique, LSI, CNRS and CEA/DSM/IRAMIS, Palaiseau F-91128, France
}

\begin{abstract}
The $3 \omega$ method is a standard way to measure the thermal conductivity of thin films. In this study, we apply the method to read the magnetic state of a perpendicularly magnetized CoTb ferrimagnetic Hall cross using a thermal excitation. In order to generate the thermal excitation, an oscillating current at an $\omega$ frequency is applied to the Hall cross using different geometries. The magnetic signals oscillating at $\omega, 2 \omega$, and $3 \omega$ are probed using a lock-in technique. From the analysis of the power dependence, we can attribute the $3 \omega$ response to the temperature oscillation and the $2 \omega$ to the temperature-gradient oscillation. Finally, the frequency dependence of the magnetic signals can be understood by considering the heat diffusion in a two-dimensional model.
\end{abstract}

\section{INTRODUCTION}

Thermal management in microelectronic devices has drawn a lot of interest, since it limits the performance of electronic devices with ever-increasing transistor density [1]. Thanks to the coupling of heat, electron, and spin, a way has been proposed to circumvent the problem by making use of the waste heat current to process information. The young research field called spincaloritronics, which stands at the convergence of two scientific fields, namely, heat transport and spintronics, is expected to deliver devices having the ability to store and/or process spin information thanks to a heat current. Thermal currents, which exist naturally in electronic devices, could then be used to measure the spin state. One important device is the nonvolatile magnetic memory for which, to read the magnetic state, an electrical current must be injected through the device. On the basis of previous work [2], we study in this paper the feasibility of thermal reading of magnetic states in Hall crosses by the oscillations of the temperature and/or temperature gradient.

Since 2008, many publications have reported magnetovoltaic signals at room temperature in response to a thermal excitation in various geometries [3-12]. While the origin of those effects, e.g., the longitudinal spin Seebeck effect (LSSE), remains under debate, the measure of voltages under an applied temperature gradient is demonstrated. Significant progress has been made for thermomagnetic effects at a high frequency or transient state. Agrawal et al. studied the LSSE of YIG/Pt in the

\footnotetext{
*daniel.lacour@univ-lorraine.fr
}

microsecond timescale [13]. Later, the frequency dependence of the LSSE up to the gigahertz range was reported through microwave heating [14]. Recently, the LSSE at several picoseconds was shown through a pump-probe method with an ultrafast heat-transfer process [15]. The underlying oscillation of the heat current plays a vital role in the high-frequency thermomagnetic effects.

In this study, we use an ac temperature method to have a higher precision and be insensitive to experimental artifacts due to thermoelectric effects [16]. The $3 \omega$ method is now a standard ac method for the measurement of the thermal conductivity of thin films [9,16-21]. If an electrical current is applied to the thin wire which oscillates at $\omega$, a temperature oscillation at $2 \omega$ is generated due to Joule heating. Given that the resistivity varies linearly with the temperature, the longitudinal resistance oscillates also at $2 \omega$. The oscillation of the voltage at $3 \omega$ (the driving current at $\omega$ times the resistance at $2 \omega$ ) can be measured synchronously with a lock-in amplifier on the thin wire. Through the measurement of the real and imaginary parts of $V_{3 \omega}$ against the frequency, the thermal conductivity of the layer beneath the metal wire can be extracted.

In this study, we extend the standard $3 \omega$ method to a Hall cross geometry by replacing the longitudinal metal resistance with the transversal resistance. Like the electrical resistivity, the Hall resistivity varies linearly with the temperature for small temperature oscillations (see Appendix B). The Hall voltage, equal to the driving current times the Hall resistivity, oscillates at $3 \omega$. Moreover, the temperature gradient is converted into an electrical field due to the spin Seebeck effect (SSE) [3,22], anomalous Nernst effect (ANE) [4,23], or Righi-Leduc 
effect (RLE) [2]. Since the temperature gradient oscillates at $2 \omega$, the information about the temperature gradient can also be extracted by measuring the signal at $2 \omega$.

To highlight the difference between the temperature oscillation and the temperature-gradient oscillation, the heat flow is generated either locally (the heating current is injected through the Hall cross) or nonlocally (the heating current is injected in a wire eccentric of the Hall cross). We first discuss the sample preparation and check the presence of a temperature gradient under local and nonlocal heating in Sec. II. The results of power dependence in local and nonlocal heating geometry are shown in Sec. III. In Sec. IV, the frequency dependences are shown and explained using a two-dimensional model. The validity of the model is verified in Sec. V. Finally, the discussion and conclusion are presented in Sec. VI.

\section{SAMPLES AND METHODS}

Hall crosses are made using a $20-\mathrm{nm} \mathrm{Co}_{76} \mathrm{~Tb}_{24}$ magnetic alloy layer. CoTb alloy is chosen because of its perpendicular magnetic anisotropy and, consequently, its square magnetization hysteresis loop when a field is applied perpendicular to the film plane [24]. The perpendicular anisotropy simplifies the magnetovoltaic response, and we consider only two possible magnetic states that could be read using a lateral temperature gradient. The CoTb layer is deposited on a glass substrate $(1 \mathrm{~mm})$ covered with a $\mathrm{Ta}(5 \mathrm{~nm}) / \mathrm{Pt}(5 \mathrm{~nm})$ buffer layer to have a smooth surface and improve the adhesion of the CoTb. A Pt $(5 \mathrm{~nm})$ capping layer is used to prevent oxidation. All layers are grown by dc magnetron cosputtering with a base pressure of less than $5 \times 10^{-9}$ mbar. After film deposition, Hall bars with dimensions $400 \times$ $200 \mu \mathrm{m}^{2}$ are patterned using UV lithography and Ar ion etching in a two-step process [Fig. 1(a)]. First, the layer is etched into a cross shape or wire shape [dark brown part in Fig. 1(a)]. After etching, electron beam evaporation of $\mathrm{Ti}(10 \mathrm{~nm}) / \mathrm{Au}(100 \mathrm{~nm})$ allows one to take contacts on the CoTb layer [brown parts in Fig. 1(a)].

The oscillating electrical current is generated by a function generator. In the local heating geometry, the electrical current is sent through the Hall cross [from contact $L$ to contact $R$, Fig. 1(a)], like in classical Hall measurements. The Joule heat is generated over the entire line. In the nonlocal heating geometry, the electrical current is sent from contact $N$ to contact $L$, generating a temperature gradient from left to right. The Hall voltage $\left[V_{+}-V_{-}\right.$ in Fig. 1(a)] is always measured between the two transversal contacts for the two geometries.

First, we study the distribution of temperature under dc Joule heating using an infrared camera. A dc current of $20 \mathrm{~mA}$ is applied either locally or nonlocally with the substrate maintained at room temperature, and the infrared images for both geometries are taken at $4.5 \mathrm{~s}$ after the current is set on [Figs. 1(c) and 1(d)]. For the local
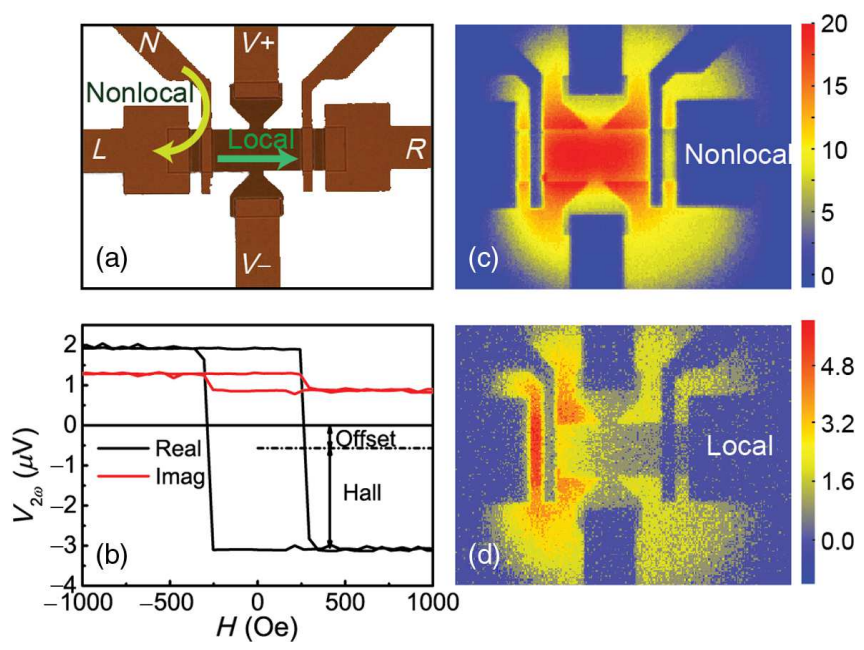

FIG. 1. (a) Optical picture of thin-film Hall cross $\mathrm{Ta}(5 \mathrm{~nm}) /$ $\operatorname{Pt}(5 \mathrm{~nm}) / \operatorname{CoTb}(20 \mathrm{~nm}) / \operatorname{Pt}(5 \mathrm{~nm})$. The voltage is measured between the two transversal contacts when an electrical current is applied locally (blue arrow) or nonlocally (yellow arrow). The three contacts used are labeled as $L, R$, and $N$. (b) The real part (black) and imaginary (red) part of the voltage at $2 \omega$ in the nonlocal geometry as a function of the external field. The current for heating is $50 \mathrm{~mA}$ at $7 \mathrm{~Hz}$. Note that a nonmagnetic contribution is present in addition to the Hall contribution. (c), (d) The temperature distributions of the Hall cross in the local geometry (c) and nonlocal geometry (d) are measured with an infrared camera at room temperature with the presence of a dc electrical current of $20 \mathrm{~mA}$. Note that the calibration of the temperature is done by measuring the infrared signal of the magnetic layer at different temperatures, and thus the temperatures for the contacts and glass are not accurate.

geometry [Fig. 1(c)], the temperature of the metal line is elevated with a maximum at the center of the line. The maximum increase of temperature at the center is estimated to be $5 \mathrm{~K}$. For the nonlocal geometry [Fig. 1(d)], the left part of the Hall cross is heated up, and we can see clearly a temperature gradient across the metal line.

In the following, an electric current of angular frequency $\omega$ is used to generate an oscillating flow of heat. The electrical signal is measured synchronously using the lockin technique. For all measurements, the reference wave is set to be in phase with the driving current $I_{0} \cos \omega t$, and the voltages at $\omega, 2 \omega$, and $3 \omega$ are recorded synchronously. The current flowing from the left contact $L$ to the right contact $R$ is labeled as $I_{x}$. Generally, the voltage can be expressed as the sum of a real part and an imaginary part: $V=V_{x}+i V_{y}$. The imaginary part of the electrical signal is zero if the voltage is induced by electrical effects, which are several orders faster than the experimental angular frequencies. For the effects related to the heat flow, a phase delay often exists between the temperature oscillation and the electrical-current oscillation.

For example, Fig. 1(b) shows the nonlocal voltage at $2 \omega$ as a function of the external field in the nonlocal geometry. 
Both real and imaginary parts depend on the magnetic state. In addition to the expected Hall effect contribution, a nonmagnetic contribution which is the average voltage of the two magnetic states arises from the small misalignment of the two transversal contacts due to the microfabrication process. Such a nonmagnetic contribution exists for all the harmonics.

For the two magnetic states, we have $V( \pm H)=V_{x}( \pm H)+$ $i V_{y}( \pm H) . V(+H)$ [respectively, $V(-H)$ ] is the Hall voltage measured for perpendicular positive (respectively, negative) saturated magnetization. Phenomenologically, we divide the signal into two contributions: a nonmagnetic contribution $V_{O}$ independent of the magnetic state and a Hall contribution $V_{H}$ that depends on the magnetic state: $V_{O / H}=\left[V_{x}(+H) \pm\right.$ $\left.V_{x}(-H) / 2\right]+i\left[V_{x}(+H) \pm V_{x}(-H) / 2\right]$. Only the Hall contribution (that we refer to as Hall voltage in the rest of the paper) is related to the magnetic effects. Note that in the following we mainly discuss the Hall contribution.

\section{POWER DEPENDENCE}

For both the local and nonlocal geometries, we present in Fig. 2 the Hall voltage at $\omega$ as a function of electricalcurrent amplitude $I_{0}$, the Hall voltage oscillation at $2 \omega$, and Hall resistance oscillation as a function of $I_{0}^{2}$. As the power of Joule heating is given by $I_{0}^{2} R$, the magnitude of the heat can be adjusted by simply controlling the electric current.

In the local geometry, the current flows from $L$ to $R$, and $I_{0}$ equals $I_{x}$. The real and imaginary parts of the Hall voltage at $\omega$ as a function of the driving current are reported in Fig. 2(a). The real part decreases linearly with the current. From the slope of the linear curve, we obtain the Hall resistance $R_{H}=0.383 \Omega$, which agrees with an independent dc measurement (not shown). The imaginary part of the Hall voltage at $\omega$ is close to zero, which indicates that the voltage at $\omega$ is purely an electrical effect and no heat process is involved.

The temperature gradient oscillates [Fig. 2(b)] at $2 \omega$ and is directly converted into a voltage at $2 \omega$ by means of the SSE, ANE, or RLE. Figure 2(b) shows the real and the imaginary parts of the Hall voltage at $2 \omega$ as a function of $I_{0}^{2}$. The linear dependence shows that the temperature gradient is also proportional to the Joule heating.

The voltage at $3 \omega$ is the oscillation of the Hall resistance at $2 \omega$ of the Hall cross times the driving current at $\omega$. Then $V_{3 \omega} / I_{0}$ corresponds to the resistance oscillation. Figure 2(c) shows the real and imaginary parts of $V_{3 \omega} / I_{0}$ (only the Hall contribution is plotted) as a function of $I_{0}^{2}$. Note that the real and imaginary parts of $V_{3 \omega} / I_{0}$ are proportional to the in-phase and out-of-phase temperature rise, respectively, because the Hall resistance is proportional to the temperature (Appendix B). $I_{0}^{2}$ can be viewed as a measure of the Joule heating. The in-phase and out-of-phase temperature oscillations increase linearly with the Joule heating.
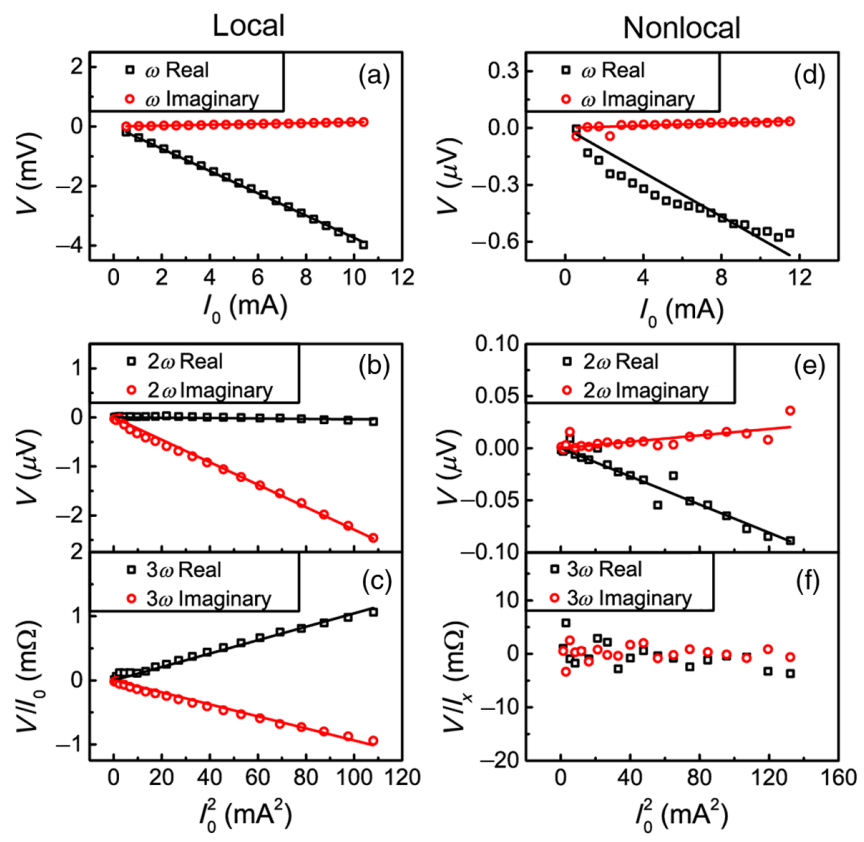

FIG. 2. Left and right columns correspond to local geometry and nonlocal geometry, respectively. Measurements are performed at $11.37 \mathrm{~Hz}$. (a),(d) Real and imaginary components of the Hall voltage measured at $\omega$ as a function of the current. (b),(e) Real and imaginary components of the Hall voltage measured at $2 \omega$ as a function of $I_{0}^{2}$. (c),(f) Real and imaginary components of the Hall contribution of $V_{3 \omega} / I$ as a function of $I_{0}^{2}$. Note, in the nonlocal geometry, $I_{0}$ is replaced by $I_{x}$, which is responsible for the Hall voltage in (d). Open symbols are experimental data, and solid lines correspond to linear fittings. The measurements are performed at $150 \mathrm{~K}$.

In the nonlocal geometry, the original idea is to generate a temperature gradient without any electrical current in the Hall cross by sending an electrical current from contact $N$ to contact $L$. Surprisingly, a leakage current still exists due to the presence of equipotential lines in the asymmetric nonlocal geometry. Figure 2(d) shows the Hall voltage measured at $\omega$ as a function of the driving current $I_{0}$. The Hall voltage is induced by a longitudinal leakage current $I_{x}$. From the Hall resistance $R_{H}$, we can deduce the longitudinal current $I_{x}=V_{\omega} / R_{H}$. $I_{x}$ in the nonlocal heating geometry is 4 orders of magnitude smaller than the nominally injected electric current $I_{0}$, which agrees with the estimation of a finite-element analysis (data not shown). The imaginary part of the Hall contribution is again close to zero, showing that the voltage at $\omega$ is independent of any thermal oscillation.

Figure 2(e) shows the voltage at $2 \omega$ as a function of $I^{2}$. The voltage, which is originating from the temperature gradient, increases linearly with the Joule heating.

Unlike the temperature-gradient oscillation, the temperature oscillation needs a probe current $I_{x}$ to measure the oscillation of the Hall resistance. The temperature gradient between the heat source and the probes needs to be taken 
into consideration to interpret the signal at higher harmonics in the nonlocal geometry. The voltage at $3 \omega$ is given by the resistance oscillating at $2 \omega$ times the longitudinal current $I_{x}$. Figure 2(f) shows the $V_{3 \omega} / I_{x}$ as a function of $I^{2}$. The strong noise indicates that the nonlocal temperature oscillation and $I_{x}$ are very weak.

\section{FREQUENCY DEPENDENCE}

The voltage at $\omega$ is independent of the temperature oscillation and remains constant for the frequency range from 3 to $200 \mathrm{~Hz}$. Since the voltages at $2 \omega$ and $3 \omega$ are related to the thermal effects, we need to consider the heat propagation to understand the frequency dependences of $V_{2 \omega}$ and $V_{3 \omega}$.

In the local geometry, the heat is liberated only through the longitudinal metal line. If we assume the heat propagates only through the glass substrate and neglect the heat propagation in the remaining metal parts, the Hall cross is simplified to be a thin metal line. Following the solution given in the literature [17], the temperature distribution induced by an infinite line is

$$
T=\frac{P}{l \pi k} e^{i 2 \omega t} K_{0}\left[\left(\frac{i 2 \omega}{\alpha}\right)^{1 / 2} r\right],
$$

where $P / l$ is the power per unit length, $\alpha$ is the thermal diffusivity, $k$ is the thermal conductivity, and $r$ is the distance to the metal heating line. The thermal diffusion length is given by $\sqrt{\alpha / i 2 \omega}$. The derivation of Eq. (1) is attached in Appendix C.

Figure 3 shows the experimental frequency dependence of $V_{3 \omega}$ in the local heating geometry. The sketch of the sample is shown in Fig. 3. The real part of $V_{3 \omega}$ clearly deviates from the linear trend in the log scale due to the large sample width. The frequency range is between 3

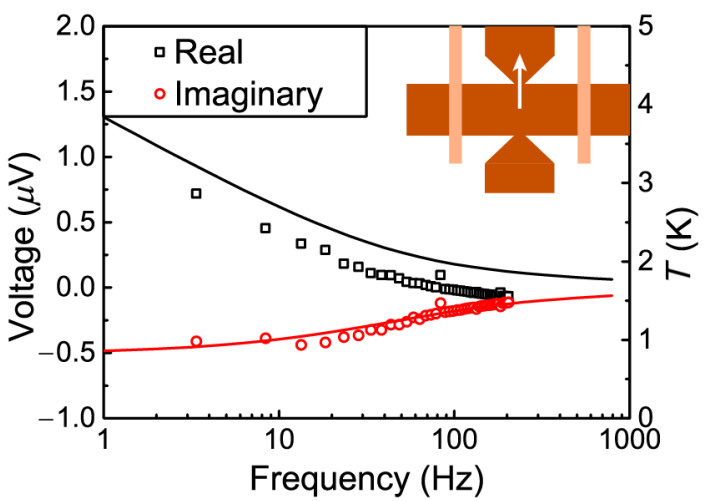

FIG. 3. Frequency dependence of the real voltage (black open circles) and imaginary voltage (red open circles) at $3 \omega$ in the local geometry at $150 \mathrm{~K}$. The solid lines correspond to the temperature oscillations calculated using the thermal diffusivity $7.8 \times 10^{-7} \mathrm{~m}^{2} / \mathrm{s}$. Inset: The white arrow shows the direction of heat flow in the Hall cross. and $200 \mathrm{~Hz}$ in this study, which corresponds to a thermal wavelength between 95 and $12 \mu \mathrm{m}$. The width between the two voltage measurement points is $200 \mu \mathrm{m}$, which is greater than the thermal wavelength. The general trend of our experimental data is reproduced using Eq. (1). The temperature as a function of the frequency shown in Fig. 3 is calculated numerically using $\alpha=7.8 \times 10^{-7} \mathrm{~m}^{2} / \mathrm{s}$, which is reported in the literature for glass at $150 \mathrm{~K}$ [25].

Figure 4 shows the frequency dependence of the voltage at $2 \omega$ in the two geometries. For the local heating geometry, the voltage induced by the longitudinal temperature gradient should be zero if the Hall cross is heated homogeneously. However, we measure a voltage independent of the frequency, which is likely due to inhomogeneous heating along the Hall bar in the local geometry. For the nonlocal heating geometry when the frequency is increased, the voltage or the temperature gradient drops quickly, since the voltage is proportional to the temperature gradient. This can be explained by the decrease of the thermal wavelength at a higher frequency. In solid lines, the temperature gradients $\partial T / \partial r$ are calculated using Eq. (1) and $\alpha=7.8 \times 10^{-7} \mathrm{~m}^{2} / \mathrm{s}$.

Note that the theoretical curves reproduce qualitatively our experimental data. Indeed, the theoretical curves are calculated considering an infinitely long heating line, which is different from the Hall cross, especially for the nonlocal geometry.

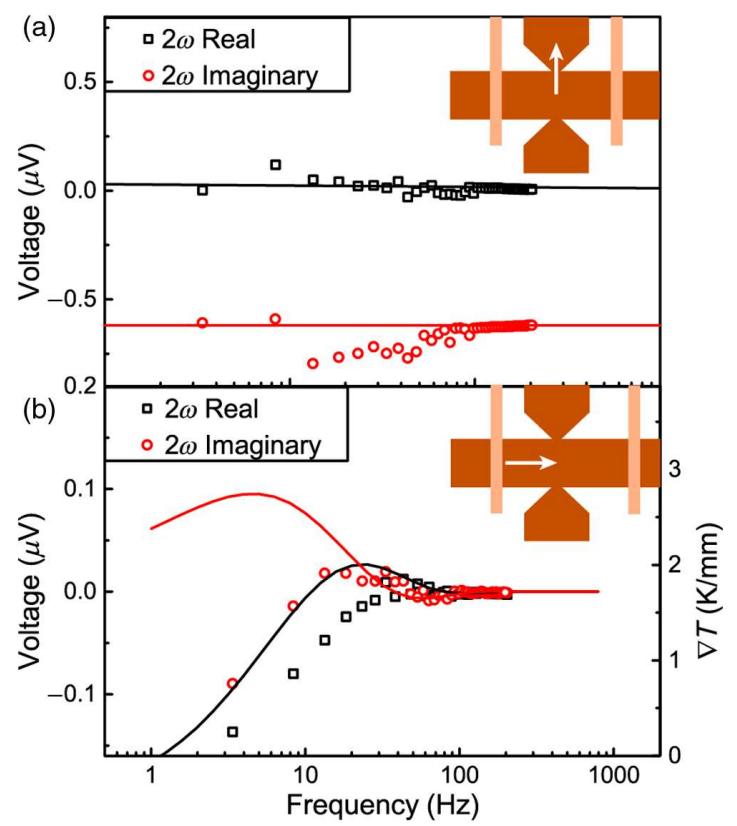

FIG. 4. The real part (black open circles) and imaginary part (red open circles) of the voltage measured at $2 \omega$ as a function of the frequency in the local (a) and the nonlocal geometry (b) at $150 \mathrm{~K}$. The solid lines in (a) are lines to guide the eye. The solid lines in (b) are the temperature-gradient oscillations calculated using $\alpha=7.8 \times 10^{-7} \mathrm{~m}^{2} / \mathrm{s}$ reported in Ref. [25]. Insets: The white arrow shows the direction of heat flow in the Hall cross for the two geometries. 


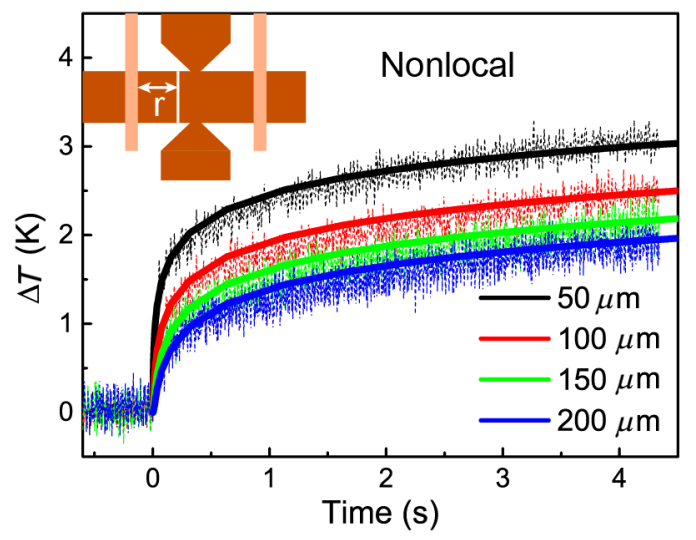

FIG. 5. The temperatures of different positions $r$ are shown as a function of the time at room temperature for the nonlocal geometry. A dc current of $20 \mathrm{~mA}$ is applied to Hall cross at $0 \mathrm{~s}$. The temperatures are extracted from the infrared images [Fig. 1(d)]. The thick solid lines correspond to the results from numerical calculations using the same model with $\alpha=6.4 \times 10^{-7} \mathrm{~m}^{2} / \mathrm{s}$. Inset: The white arrow shows the direction of heat flow in the Hall cross.

\section{TIME-RESOLVED TEMPERATURE MEASUREMENT}

To further test the validity of the model for the Hall cross, we apply the model to the time-resolved temperature measurement in the nonlocal geometry with an infrared camera. We measure the temperature evolution of the Hall cross after a dc heating current using a time-resolved infrared camera at room temperature. Figure 5 shows the temperature evolution for different positions on the Hall cross as a function of the time in the nonlocal geometry. The final temperature distribution of the whole Hall cross after $4.5 \mathrm{~s}$ is shown in Fig. 1(d). As the distance with respect to the heat source increases, the temperature increases more slowly. The temperature evolution is calculated numerically with the model under a Heaviside step function to simulate the input heat power of $20 \mathrm{~mA} \mathrm{dc}$ current. The fitting is performed using $\alpha=6.4 \times 10^{-7} \mathrm{~m}^{2} / \mathrm{s}$ at room temperature, which is reported in the literature [25]. The agreement between the measured and modeled temperature profiles at different locations in the Hall cross during the transient stage confirms the validity of our thermal model.

\section{DISCUSSION AND CONCLUSION}

Our study raises several issues on the use of a combined $3 \omega$ and Hall measurement to read the magnetic configuration. In order to highlight those issues and improve the measurement, we provide the following clarifications:

(1) A leakage electric current is detected in the nonlocal geometry. The longitudinal component of this current $I_{x}$ could be estimated to be about $0.1 \%$ of the injected longitudinal current in the local geometry.
However, the heat dissipated by the leakage current (proportional to $I_{x}^{2}$ ) is negligible. As a result, we can conclude that this leakage current does not affect the temperature gradient in the nonlocal geometry.

(2) The Hall voltage at $3 \omega$ is given by the driving current times the oscillating anomalous Hall resistance. Consequently, a design with a greater amplitude of the anomalous Hall resistance would improve the $3 \omega$ output signal.

(3) The physical origin of the $2 \omega$ signal cannot be identified by our analysis of the temperature oscillation and temperature-gradient oscillation. Indeed, to our knowledge, the longitudinal temperature along the Hall bar can be converted into a transversal voltage by the SSE, ANE, or RLE. Several methods are proposed in the literature to identify the origins [2,26-28].

(4) Since the thermal wavelength is critical in the heatrelated phenomenon, the probe should be shorter than the thermal wavelength to measure the magnetic states with an oscillating heat current. This is always satisfied in the local geometry, as we are probing at a heated spot. In the nonlocal geometry, the amplitude of the temperature oscillation decays at the scale of a thermal wavelength. For a given frequency, the distance between the probe and the source should as small as possible. One could improve the signal by making the probe distance smaller or at least comparable to the thermal wavelength.

(5) In an optimized device, the substrate should have a high thermal diffusivity and a low electric conductivity. High thermal diffusivity means a higher thermal wavelength, which is beneficial for the probe of magnetic states. An electrically conductive substrate may bring spurious effects reported before in ac measurements $[10,12]$.

Based on the analyses of the $3 \omega$ method, we show that it is possible to read the magnetic states using heat $\left(V_{2 \omega}\right)$. In the thermal point of view, we give here the key ingredients to optimize the sample design: (i) The distance between the probe and heat source should be smaller than the thermal wavelength of the working frequency, or, equivalently, the thermal reading should be performed below the cutoff frequency for a given Hall cross. (ii) Substrates with a high thermal diffusivity will be preferable. (iii) Further improvement of the signal needs the clarification and enhancement of the physical mechanisms, which include the SSE, ANE, and RLE.

In conclusion, the $3 \omega$ method is combined with the anomalous Hall voltage measurement to read the magnetic configuration of $\mathrm{CoTb}$ in a Hall cross using an oscillating thermal excitation. From the power dependence and frequency dependence, we identify that the voltage responses $V_{\omega}, V_{2 \omega}$, and $V_{3 \omega}$ come from the 
electrical current, temperature-gradient, and temperature oscillations, respectively. This method can be generalized to Hall crosses based on other materials.

\section{ACKNOWLEDGMENTS}

The authors thank Yuan Lu for fruitful discussions and Guillaume Sala for help with the experiments. This work was supported by the Carnot ICEEL Flamme project. Experiments were performed using equipment from the TUBE-Daum funded by FEDER (EU), ANR, the Region Lorraine, and Grand Nancy. This work was supported partly by the French PIA project "Lorraine Universite d'Excellence," Reference No. ANR-15-IDEX-04-LUE.

\section{APPENDIX A: NONMAGNETIC CONTRIBUTION IN THE LOCAL GEOMETRY AND NONLOCAL GEOMETRY}

In addition to the Hall contribution, a nonmagnetic contribution presents for all the harmonics. The nonmagnetic part contributes to the $3 \omega$ signal as is discussed extensively by previous publications about the 3-omega method [17,21]. Figure 6 displays the power dependence of $V_{2 \omega}$ and $V_{3 \omega}$ in the local and nonlocal geometry.

The nonmagnetic contribution mainly comes from the imperfections of the Hall cross, such as misalignment of the electrodes and inhomogeneity of the film thickness. We can observe an unexpected behavior at a low heating current. We attribute the artifact to the preamplifier, which generates a weak higher-harmonic signal.
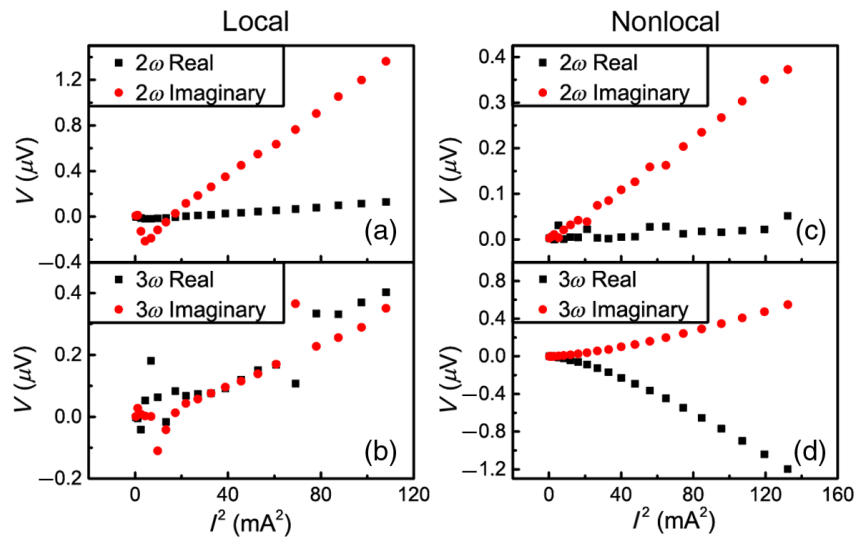

FIG. 6. In the left column, real and imaginary components of the nonmagnetic voltage measured at $2 \omega$ (a) and $3 \omega$ (b) are plotted as a function of $I^{2}$ in the local geometry. In the right column, real and imaginary components of the voltage at $2 \omega$ (c) and $3 \omega(\mathrm{d})$ are presented as a function of $I^{2}$ in the nonlocal geometry.

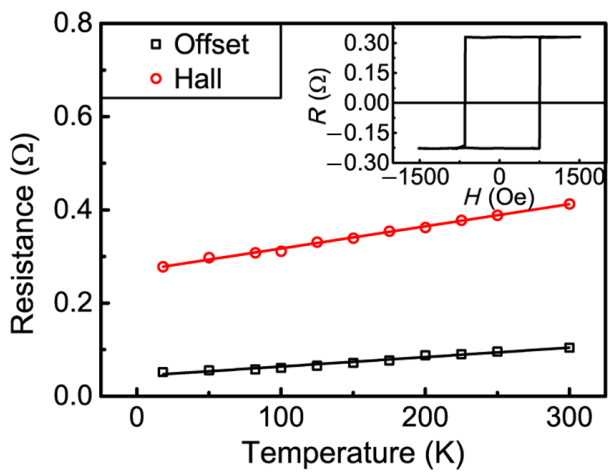

FIG. 7. Offset resistance (open square) and Hall resistance (open circle) are plotted as a function of the temperature. The solid lines correspond to the linear fittings. For each temperature, the offset resistance and Hall resistance are extracted from a hysteresis loop. Inset: Hall resistance $R$ at $18 \mathrm{~K}$ is plotted as a function of external field $H$. A constant electric current of $1 \mathrm{~mA}$ is used for the measurements.

\section{APPENDIX B: ANOMALOUS HALL RESISTANCE AS A FUNCTION OF TEMPERATURE}

Figure 7 shows the offset resistance and anomalous Hall resistance as a function of the temperature for one Hall cross. It is nearly linear between the range of 18 and $300 \mathrm{~K}$. For a small temperature oscillation $(<5 \mathrm{~K})$, we can consider that the resistance is proportional to the temperature. The slope used for the fitting is $4.76 \times 10^{-4} \Omega / \mathrm{K}$.

\section{APPENDIX C: DERIVATION OF EQ. (1)}

The derivation of Eq. (1) is given in the book of Carslaw and Jaeger [29]. For the sake of clarity, we attach the derivation of Eq. (1) in this section. The general heat equation reads

$$
\rho c_{p} \frac{\partial T}{\partial t}-\boldsymbol{\nabla} \cdot(k \nabla T)=\dot{q}
$$

where $\rho$ is the mass density, $c_{p}$ the specific heat capacity, $k$ the thermal conductivity, and $\dot{q}$ the heat source.

An instantaneous point source releases a quantity of heat $\rho c_{p}$ at $t=0$ in an infinite three-dimensional space; the temperature distribution is given by

$$
T(r, t)=\frac{1}{8(\pi \alpha t)^{3 / 2}} e^{-r^{2} / 4 \alpha t}
$$

where $\alpha=k / \rho c_{p}$ and $r$ is the distance to the point source. Consider a thin infinity long heat line in an infinite threedimensional space and a quantity of heat per unit length of line $\rho c_{p}$ liberated from the line at $t=0$; the temperature distribution is obtained by integrating the point source solution over the line: 


$$
T(r, t)=\frac{1}{4 \pi \alpha t} e^{-r^{2} / 4 \alpha t} .
$$

If the heat is released periodically at $\rho c_{p} e^{i 2 \omega t}$ per unit length, the temperature distribution is given by integrating the solution of the infinite long line source over time from $-\infty$ to $t$ :

$$
\begin{aligned}
T(r, t) & =\frac{1}{4 \pi \alpha} \int_{-\infty}^{t} e^{i 2 \omega t^{\prime}} e^{-r^{2} /\left[4 \alpha\left(t-t^{\prime}\right)\right]} \frac{d t^{\prime}}{t-t^{\prime}} \\
& =\frac{1}{2 \pi \alpha} e^{i 2 \omega t} K_{0}\left[\left(\frac{i 2 \omega}{\alpha}\right)^{1 / 2} r\right] .
\end{aligned}
$$

A factor of 2 should be added when we apply the equation to the wire-on-substrate structure, where the substrate is assumed to be semi-infinite.

[1] P. K. Schelling, L. Shi, and K. E. Goodson, Managing heat for electronics, Mater. Today 8, 30 (2005).

[2] B. Madon, D. C. Pham, J.-E. Wegrowe, D. Lacour, M. Hehn, V. Polewczyk, A. Anane, and V. Cros, Anomalous and planar Righi-Leduc effects in $\mathrm{Ni}_{80} \mathrm{Fe}_{20}$ ferromagnets, Phys. Rev. B 94, 144423 (2016).

[3] K. Uchida, S. Takahashi, K. Harii, J. Ieda, W. Koshibae, K. Ando, S. Maekawa, and E. Saitoh, Observation of the spin Seebeck effect, Nature (London) 455, 778 (2008).

[4] A. D. Avery, M. R. Pufall, and B. L. Zink, Observation of the Planar Nernst Effect in Permalloy and Nickel Thin Films with In-Plane Thermal Gradients, Phys. Rev. Lett. 109, 196602 (2012).

[5] G. E. W. Bauer, E. Saitoh, and B. J. van Wees, Spin caloritronics, Nat. Mater. 11, 391 (2012).

[6] S. Y. Huang, X. Fan, D. Qu, Y. P. Chen, W. G. Wang, J. Wu, T. Y. Chen, J. Q. Xiao, and C. L. Chien, Transport Magnetic Proximity Effects in Platinum, Phys. Rev. Lett. 109, 107204 (2012).

[7] M. Schmid, S. Srichandan, D. Meier, T. Kuschel, J.-M. Schmalhorst, M. Vogel, G. Reiss, C. Strunk, and C. H. Back, Transverse Spin Seebeck Effect versus Anomalous and Planar Nernst Effects in Permalloy Thin Films, Phys. Rev. Lett. 111, 187201 (2013).

[8] J. Sinova, Spin Seebeck effect: Thinks globally but acts locally, Nat. Mater. 9, 880 (2010).

[9] J.-E. Wegrowe, H.-J. Drouhin, and D. Lacour, Anisotropic magnetothermal transport and spin Seebeck effect, Phys. Rev. B 89, 094409 (2014).

[10] Y. Xu, W. Lin, S. Petit-Watelot, M. Hehn, H. Rinnert, Y. Lu, F. Montaigne, D. Lacour, S. Andrieu, and S. Mangin, Origins of large light induced voltage in magnetic tunnel junctions grown on semiconductor substrates, J. Appl. Phys. 119, 023907 (2016).

[11] W. Lin, M. Hehn, L. Chaput, B. Negulescu, S. Andrieu, F. Montaigne, and S. Mangin, Giant spin-dependent thermoelectric effect in magnetic tunnel junctions, Nat. Commun. 3, 744 (2012).
[12] A. Boehnke, M. Walter, N. Roschewsky, T. Eggebrecht, V. Drewello, K. Rott, M. Münzenberg, A. Thomas, and G. Reiss, Time-resolved measurement of the tunnel magnetoSeebeck effect in a single magnetic tunnel junction, Rev. Sci. Instrum. 84, 063905 (2013).

[13] M. Agrawal, V. I. Vasyuchka, A. A. Serga, A. Kirihara, P. Pirro, T. Langner, M. B. Jungfleisch, A. V. Chumak, E. T. Papaioannou, and B. Hillebrands, Role of bulk-magnon transport in the temporal evolution of the longitudinal spinSeebeck effect, Phys. Rev. B 89, 224414 (2014).

[14] M. Schreier, F. Kramer, H. Huebl, S. Geprägs, R. Gross, S. T. B. Goennenwein, T. Noack, T. Langner, A. A. Serga, B. Hillebrands, and V. I. Vasyuchka, Spin Seebeck effect at microwave frequencies, Phys. Rev. B 93, 224430 (2016).

[15] J. Kimling, G.-M. Choi, J. T. Brangham, T. Matalla-Wagner, T. Huebner, T. Kuschel, F. Yang, and D. G. Cahill, Picosecond Spin Seebeck Effect, Phys. Rev. Lett. 118, 057201 (2017).

[16] C. Dames, Measuring the thermal conductivity of thin films: 3 Omega and related electrothermal methods, Annual review of heat transfer 16, 7 (2013).

[17] D. G. Cahill, Thermal conductivity measurement from 30 to $750 \mathrm{~K}$ : The $3 \omega$ method, Rev. Sci. Instrum. 61, 802 (1990).

[18] D. G. Cahill, W. K. Ford, K. E. Goodson, G. D. Mahan, A. Majumdar, H. J. Maris, R. Merlin, and S. R. Phillpot, Nanoscale thermal transport, J. Appl. Phys. 93, 793 (2003).

[19] D. G. Cahill, P. V. Braun, G. Chen, D. R. Clarke, S. Fan, K. E. Goodson, P. Keblinski, W. P. King, G. D. Mahan, A. Majumdar, H. J. Maris, S. R. Phillpot, E. Pop, and L. Shi, Nanoscale thermal transport. II. 2003-2012, Appl. Phys. Rev. 1, 011305 (2014).

[20] J. Kimling, J. Gooth, and K. Nielsch, Anisotropic magnetothermal resistance in Ni nanowires, Phys. Rev. B 87, 094409 (2013).

[21] C. Dames and G. Chen, $1 \omega, 2 \omega$, and $3 \omega$ methods for measurements of thermal properties, Rev. Sci. Instrum. 76, 124902 (2005).

[22] M. Schreier, N. Roschewsky, E. Dobler, S. Meyer, H. Huebl, R. Gross, and S. T. B. Goennenwein, Current heating induced spin Seebeck effect, Appl. Phys. Lett. 103, 242404 (2013).

[23] M. Mizuguchi, S. Ohata, K. Uchida, E. Saitoh, and K. Takanashi, Anomalous Nernst effect in an $L 1_{0}$-ordered epitaxial FePt thin film, Appl. Phys. Express 5, 093002 (2012).

[24] M. Gottwald, M. Hehn, F. Montaigne, D. Lacour, G. Lengaigne, S. Suire, and S. Mangin, Magnetoresistive effects in perpendicularly magnetized Tb-Co alloy based thin films and spin valves, J. Appl. Phys. 111, 083904 (2012).

[25] G. Yang, A. D. Migone, and K. W. Johnson, Heat capacity and thermal diffusivity of a glass sample, Phys. Rev. B 45, 157 (1992).

[26] S. M. Wu, J. Hoffman, J. E. Pearson, and A. Bhattacharya, Unambiguous separation of the inverse spin Hall and anomalous Nernst effects within a ferromagnetic metal 
using the spin Seebeck effect, Appl. Phys. Lett. 105, 092409 (2014).

[27] D. Tian, Y. Li, D. Qu, X. Jin, and C. L. Chien, Separation of spin Seebeck effect and anomalous Nernst effect in Co/Cu/YIG, Appl. Phys. Lett. 106, 212407 (2015).
[28] S. H. Wang, L. K. Zou, J. W. Cai, B. G. Shen, and J. R. Sun, Transverse thermoelectric effects in platinum strips on permalloy films, Phys. Rev. B 88, 214304 (2013).

[29] H. S. Carslaw and J. C. Jaeger, Conduction of Heat in Solids (Oxford University, New York, 1986). 